\title{
MACHINE LEARNING TO SUPPORT INDUSTRIAL DIGITALIZATION AND BUSINESS TRANSFORMATION
}

\author{
Agostino G. Bruzzone ${ }^{(a)}$, Marina Massei ${ }^{(b)}$, Kirill Sinelshchikov $^{(\mathrm{c})}$, \\ Giuliano Fabbrini $^{(\mathrm{d})}$, Marco Gotelli ${ }^{(\mathrm{e})}$, Alberto Molinari ${ }^{(\mathrm{f})}$ \\ (a), (b), (c) Simulation Team, Genoa University \\ (d), (e) SIM4Future

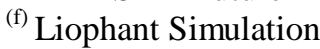 \\ (a), (b), (c) \{agostino.bruzzone, marina.massei, kirill\}@ simulationteam.com \\ (d), (e) \{ giuliano.fabbrini, marco.gotelli\} @ sim4future.com \\ (f) alberto.molinari@liophant.org
}

\begin{abstract}
This paper addresses use of Artificial Intelligence (AI) and in particular Intelligent Agents (IA) in order to evaluate efficiency of information exchange and awareness in Small and Medium Enterprise (SME), with particular attention to digital transformation. To perform required experimentation, the authors have developed a Serious Game (SG) named JANUS, in which the player interacts with intelligent agents representing a virtual company and its actions aim to acquire as much as possible data about the organization.
\end{abstract}

Keywords: Modeling, Simulation, AI, IA, ANN, Data analytics, CPM, CRM, Digitalization

\section{INTRODUCTION}

Given the evolution in business processes as well as the rising of technological enablers, digital transformation is one of the main trends in development of modern business along these day. For instance, modern software packages and solutions are used to support activities for a variety of different fields and industries, such as supply chain management, customer management, organization support, production, service and maintenance.

Based on the state-of-the-art, it is confirmed that data analytics and business intelligence are some of the main pillars of digital transformation because allows to provide valuable information to the people to support decisions as well as guide effective management. At the same time, particular attention should be focused on aspects such as digital marketing operations and corporate performance management. Hence, these sectors are very promising in terms of potential business improvement through innovative solutions able to react promptly to situation evolution and to capture expectations and behaviors of existing and potential customers. All these potential achievements are strongly related, not only to the capability to properly redesign business processes, but also to human resources attitudes and capabilities and from this point of view it is evident the necessity to develop the cultural background in the companies to get full advantage of these technologies. At the same time there is a significant need for introducing new people and to train them to become familiar with the concepts as well as with the software solutions and new organizational processes.

\section{NEEDS AND CHALLENGES}

Due to these reasons, it was decided to develop a serious game devoted to test ability of a young engineer or consultant to face the challenge of a transformation project within a company and to study the potential of this kind of application as well as the different further development that could be enabled by integrating it with Intelligent Agents. So this study focuses on the different approaches devoted to design and deliver new solution enabling cutting-edge services based on available big data and related capabilities in checking, validating and mining them. This opportunity (innovative business models and management) is very attractive to industries, but obviously requires a clear understanding of capabilities, constraints and critical issues in introducing, activating and using modern integrated solutions such as CRM (Customer Relationship Management) and CPM (Corporate Performance Management) (Bohling et al. 2006). Indeed, a crucial aspect identified during the business experiences matching with research results, it was that digital transformation and big data analysis allow both redesigning business models and change also the roles of those working in business analysis and business management. Obviously, introducing systems which integrate company information may cause new possible vulnerability and point of failure. Indeed, if compromising a single computer typically was not an issue for stand-alone systems, for today's integrated systems the risk to lost all information at once creates a lot of concerns in companies (Bruzzone, 2017b). 


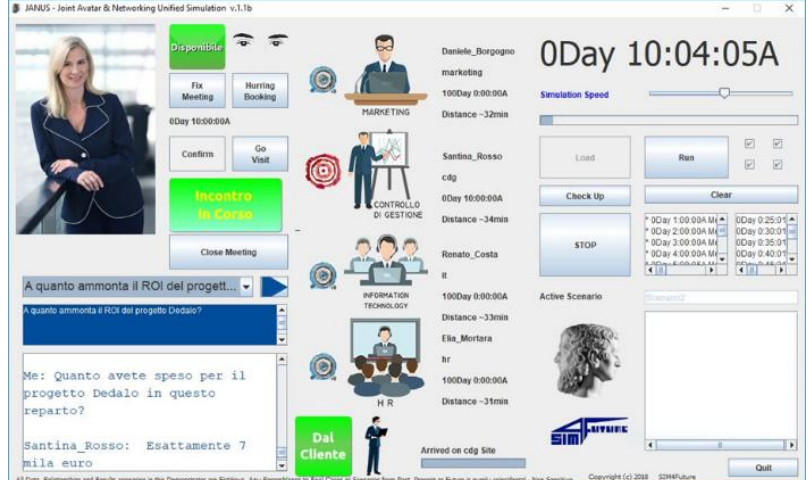

Figure 1: Janus Simulation

\section{DIGITAL TRANSFORMATION PROJECTS}

It was decided to consider the transformation projects as the mission environments to be used to develop this new capability and the scenario for the serious games. Among the different ones, considering current business development and in reference to previously mentioned aspect the focus was on transformation initiatives based on adoption of CPM and CRM solutions. Indeed, these kind of systems are expected to be able to improve the efficiency of company management and reactivity to external and internal stimulations; therefore to achieve these results it is fundamental to properly develop, implement and use these tools and it is not rare to observe projects of transformation that fall into a deadlock or that don't perform as expected. Due to these reasons the use of serious games to train, evaluate and even learn new correlations on resources involved in these initiatives resulted very interesting. Indeed, the spectrum of cases is pretty wide; in some case the companies do not have the resources (in terms of finance or people) to guarantee the possibility to introduce new systems all over the processes, hence, they keep using "legacy" applications, sometime quite obsolete; otherwise spreadsheets are extremely popular and result often the preferred office package to support business processes with all flexibility advantages, but also strong limitations for common work, reliability, reuse, sharing, updates, etc; these situations are quite typical for small companies, but also big companies are afflicted by these cases with the additional aspect to have a galaxy of different solutions with very limited and "unreliable" integration (Krumbholz et al. 2001; Gérpm 2017). In some other cases, problems are related to improper systems configuration and use, which is often caused by not skilled, or not motivated, personnel, which result often into not being capable to benefit from the potential of valuable solutions. Among the multiple critical issue, it should be mentioned, that the development and implementation team often are not very well amalgamated and could have different specific goals that tend to increase project duration, unnecessary customization, twisted processes and so on. In any case, in order to improve overall efficiency in one of this projects, it is necessary to understand quickly all possible pitfalls of the business process, define where and how to introduce digitalization and check what kind of risks are related to the introduction and utilization of new systems considering both ICT, business and human factors (Bruzzone et al. 2004).

\section{JANUS}

Due to the above mentioned issue the Simulation Team have developed JANUS (Joint Avatar \& Networking Unified Simulation), a discreet event stochastic engine acting as a Serious Game capable to reproduce human behaviors and reactions. JANUS is specifically designed to be adopted in scenarios related to business digitalization. In order to improve involvement and motivation of the players it was decided to adopt the MS2G approach (Modeling, interoperable Simulation and Serious Games) that guarantee the engagement of user and modularity of the models (Bruzzone, 2018; De Gloria et al., 2012). In fact, the creation of interesting and immersive scenarios allows to achieve multiple objectives, for instance to acquire more data by stimulating player to perform more simulation runs; in this way it is possible to use the serious games not only for training of people assessment, but also to capture emergent tactics and effective playing strategies by the multiple users based on crowdsourcing approach (Massei et al. 2014; Bruzzone et al.2017c). In JANUS, it was created a set of predefined data for basic playing, therefore, the system is fully open to be further integrated with other models and data, modifying information accordingly to the parallel evolution of the scenario thanks to the flexibility of the proposed interoperable models. Indeed, JANUS is a Serious Game (SG) devoted to address multiple business and consulting issues including among the others virtual interview with avatars controlled by Intelligent Agents (IA), as well as time management and meeting scheduling considering human behavior modifiers (HBM). In this case study, the intelligent agents represent key figures of a company (i.e. managers of different divisions) that play their specific roles while demonstrating their individuality due to their cultural heritage or background (Bruzzone et al., 2015; Bruzzone, 2017a). Indeed, every avatar is characterized by a set of individual characteristics including among the others: cultural, educational and professional background, as well as social network in the company; in addition the avatar is characterized by its openness to change, hostility to consultants, smartness, laziness, punctuality, precision, aggressiveness, stress, fatigue, fear, reliability and honesty. The use of intelligent agents as sparring partners allows not only to challenge the player, but also to improve the quality of interactions during the game, making the entire experimentation immersive and engaging (Bruzzone et al., 2014). As demonstration of this fact, the authors observing neutrally the users at "play" with JANUES collected evidence of emotional engagement by laughing, snorting and swearing at the computer based on the IA reactions. In facts, the JANUS scenario evolves in time as well as based on actions of the player. For example, delays on meetings or improper 
behaviors influence the avatars and make them more hostile and less available in providing reliable and precise feedback and values on the company's Key Performance Indicators (KPI). This may reach extreme outcomes (i.e. avatars refuse the meeting or lie to the human player). By the way the incorrect data provided by avatar could be identified by data mining different virtual managers reports and simulated project evolution during the game by smart players that pay attention to the general situation. Indeed, player's actions impact on the perception of the avatar, which could become friendly and open or hostile and aggressive. In order to conduct the experimentation, it was selected a set of professionals working in the field of interest, mostly from consulting companies, as well as university students in management and engineering. During the experimentation, these players had the possibility to run multiple time JANUS analyzing each time a different scenario generated stochastically by the game, with different virtual managers and digitalization projects. For each run it was possible to collect logs and data regarding the evolution of the scenario and the state of the virtual environment, such as company's data, avatars' parameters, players' interactions, etc. The application logs have been collected, filtered and analyzed in order to assess also players' skills in terms of evaluation capability in various cases, such as in with or without presence of new valid CPM and CRM solutions. In order to analyze experimental results, the authors have decided to developed an Artificial Neural Network, (ANN) able to assess the performance enhancement caused by using the CRM/CPM solutions on the specific digitalization project and, at the same time, discriminate various capabilities of human players based on their interviews with avatars. Indeed, each player has to plan the interviews with avatars, develop time schedule for visiting offices and departments avoiding delays, time slot overlapping and, finally, conduct interviews selecting available questions (fig.1)

\subsection{IMPLEMENTATION DETAILS}

From the beginning of the study, it was planned to involve large number of people in the experimentation phase; typically, in such situations participants use different versions of distinct operation systems. To guarantee portability, flexibility easy use and compatibility issues, the authors have adopted Java programming language to make the Serious Game platform independent; indeed even the Graphic User Interface (GUI) is implemented in simple, but reliable, way by Java Swing toolkit. Given this architecture, many professional consultants, as well as students within these fields of interest, were able to be easily involved in the experimentation and to provide the authors with sufficient data sets for analysis.

\section{DATA ANALYTICS AND AI}

Obviously, there are numerous possibilities to analyze experimental data, starting from basic statistical analysis up to artificial intelligence; often, it is convenient to adopt more than one (Montgomery, 2017). Indeed, the authors have used different techniques for different datasets; for instance, the duration of tests and percentage of errors performed by the players was compared based on their statistical distribution. At the same time, the performance of the virtual company and avatars, in the different scenarios (with or without information management solutions), was assessed using ANN (Artificial Neural Network).

Obviously the proposed results in this paper represents only a preliminary demonstration of a potential ability to develop a support system for assessment sessions, meetings and interviews with experts. In order to perform data classification, the authors have developed an application using Tensorflow library for Python.

According to the number of variables to be taken into account and number of possible categories for classification, input and output layers of the neural network have been experimented with 14 and 4 neurons each. At the same time, a specific analysis was carried out to identify the optimal numbers of neurons in the hidden layer for this case and experimentally it resulted to be equal to 20 .

Furthermore, the authors tested different types of activation functions and type of gradient based optimization, finding that in the case of interest Hyperbolic Tangent Activation Function (tanh) and Root Mean Square Propagation (RMSProp) are most suitable candidates (Géron, 2017). It is possible to state that developed ANN allowed to achieve almost 96\% precision in classification after 1000 cycles of training just over the testing data set.

\section{MODELS AND PLAYING MODES}

As mentioned, in order to perform experimentation, the authors have developed multiple scenarios, characterized by different sets of parameters to be identified, possible questions and answers as well as behavior of the avatars.

The players are able to plan meeting with the departments' representatives in order to interview them and collect the desired information. Due to the stochastic nature of the simulation, the participant could face partial or complete overlapping of meetings times, as proposed by the avatars, hence, being forced to choose between delays or postponing some meetings. Obviously, in such circumstances, some avatars could become less friendly to the user, or even hostile. Another possibility to face unfriendly behavior is to arrange multiple meetings with the same persons, each time obtaining only partial information.

Based on the perception of the avatar, the dialog options could differ, while provided information could be more or less precise. Indeed, the participants were required to run the simulation multiple times, performing required set of interviews with IA and indicating their guesses about values of the parameters of interest at the end of each run; data acquired at each step of the game was saved in dedicated log files to be consequently analyzed by the authors. 


\section{RESULTS}

By applying ANN to the data from the log files, it was confirmed a strong correlation between level of digitalization of the virtual company and error level made by the player while guessing parameters values during the simulation; the precision of estimation highly depends on such systems as CPM and CRM. As anticipated, the ANN is capable to identify presence of new reliable software solutions with precision almost 96\% (this based only on the estimation of error values) confirming importance of employment of such systems in companies. Another interesting data was obtained by analyzing logs corresponding to multiple sequential simulation runs performed by the same player. Indeed, while large part of the participants conducted only 2 simulations, many players did 4 or more runs. In such cases it was found that precision of human players typically demonstrates notable improvement after the second run, when estimation error of correct values decreases by almost $20 \%$. More significant difference is observed in case of comparison of simulation duration. In this case, typical duration of runs decreased by more than $50 \%$ after the 3rd tentative, which is likely related to the familiarization with the software.

\section{CONCLUSIONS}

The proposed approach made possible to finalize a general analysis on digitalization solutions and methodologies in relation with actual case studies.

A simulation engine has been developed and implemented into a serious game able to engage users into challenging scenarios related to company digitalization projects.

In facts, the experimentation conducted with professionals from consulting companies, led to validate the system for this operating context.

Therefore just preliminary results of the experimentation carried out on these models is proposed within this paper, therefore the authors are active in conducting additional tests and experimentations as well as extending the simulation engine and IA capabilities. A basic artificial neural network has been developed, then its ability to learn correlations and classify the different cases has been assessed, evaluating its generalization capacity. These experimental results are obviously only a starting point, a demonstration of a potential capacity which, based on the results, looks promising. In the future, the author expect also to further develop similar games to be applied extensively in consulting and digitalization projects to support actors in this field and to assess the skills of potential new resources.

\section{REFERENCES}

Bohling, T., Bowman, D., LaValle, S., Mittal, V., Narayandas, D., Ramani, G., \& Varadarajan, R. (2006). CRM implementation: Effectiveness issues and insights. Journal of Service Research, 9(2), 184-194.
Bruzzone A.G. (2018). MS2G as pillar for developing strategic engineering as a new discipline for complex problem solving. In proceedings of 30th European Modeling and Simulation Symposium, EMSS 2018 pp. 405-411.

Bruzzone A.G. (2017a). Smart Simulation: Intelligent Agents, Simulation and Serious Games as enablers for Creating New Solutions in Engineering, Industry and Service of the Society. Keynote Speech at International Top-level Forum on Engineering Science and Technology Development Strategy- Artificial intelligence and simulation, Hangzhou, China.

Bruzzone, A.G. (2017b). Information Security: Threats \& Opportunities in a Safeguarding Perspective. Keynote speech at World Engineering Forum, Rome, Italy.

Bruzzone, A. G., \& Massei, M. (2017c). Simulationbased military training. In Guide to SimulationBased Disciplines (pp. 315-361). Springer, Cham.

Bruzzone A.G., Massei M., Poggi S., Bartolucci C., Ferrando A.(2015) Intelligent agents for human behavior modeling as support to operation. Simulation and Modeling Methodologies, Technologies and Applications, Springer, pp. 119132.

Bruzzone A.G., Massei, M., Longo, F., Poggi, S., Agresta, M., Bartolucci, C., \& Nicoletti, L. (2014). Human Behavior Simulation for Complex Scenarios based on Intelligent Agents. Proceedings of the 2014 Annual Simulation Symposium, SCS, San Diego, CA, April.

Bruzzone, A. G., Viazzo, S., Briano, C., \& Massei, M. (2004). Modelling Human Behaviour in Industrial Facilities \& Business Processes. SIMULATION SERIES, 36(2), 15.

De Gloria, A., Bellotti, F., \& Berta, R. (2012). Building a comprehensive $R \& D$ community on serious games. Procedia Computer Science, 15, 1-3.

Gargeya, V. B., \& Brady, C. (2005). Success and failure factors of adopting SAP in ERP system implementation. Business process management journal, 11(5), 501-516.

Géron, A. (2017). Hands-on machine learning with Scikit-Learn and TensorFlow: concepts, tools, and techniques to build intelligent systems. "O'Reilly Media, Inc.".

Krumbholz, M., \& Maiden, N. (2001). The implementation of enterprise resource planning packages in different organisational and national cultures. Information systems, 26(3), 185-204.

Massei, M., Poggi, S., Agresta, M., \& Ferrando, A. (2014). Development planning based on interoperable agent driven simulation. Journal of Computational Science, 5(3), 395-407.

Montgomery, D. C. (2017). Design and analysis of experiments. John Wiley \& Sons, NYC. 